9. Сивцев, П. В. Численное исследование некоторых прикладных проблем расчета напряженно-деформированного состояния / П. В. Сивцев // Диссертация, Якутск, Северовосточный федеральный ун-т им. М.К. Амосова. - 2018. - 148 с.

10. Сегерлинд, Л. Применение метода конечных элементов / Л. Сегерлинд. - М.: Мир, 1979. -392 c.

Надійшла до редколегї 30.10.2019

УДК 669.162.266.242:

669.102.267.34.001.57

\author{
В. И. Елисеев ${ }^{1}$, А. П. Толстопят ${ }^{1}$, Л. А. Флеер ${ }^{1}$, \\ Ю. П. Совит ${ }^{1}$, А. Ф. Шевченко ${ }^{2}$, С. А. Шевченко ${ }^{2}$ \\ ${ }^{1}$ Днепровский национальный университет имени Олеся Гончара \\ ${ }^{2}$ Институт черной металлургии НАНУ, г. Днепр
}

\title{
ДВИЖЕНИЕ ДВУХФАЗНОГО ПОТОКА В ФУРМЕ
}

На основе теории двухфазных течений (газ - твердые частицы) рассмотрена задача движения потоков в производственных трассах для подачи сыпучего магния в расплав чугуна. Данная работа является в какой-то степени подытоживающей некоторый этап лабораторных экспериментальных исследований, исследований в промышленных условиях, а также теоретических разработок. Несмотря на большие успехи и широкое использование теории взаимопроникающих сред, являющейся основной идеей подхода к расчету многофазных течений и установления необходимых зависимостей при взаимодействии фаз, важным условием остается вопрос о силовом воздействии стенок канала на движущиеся частицы. Имеющиеся литературные данные позволяют делать оценки нужных параметров, однако для длинных производственных трасс с изменяющимися геометрическими характеристиками не снимается необходиость экспериментального определения этих параметров в конкретных условиях. Такие данные были получены в результате многолетней работы на произвдственных площадках Китая, они вошли в соответствующие коэффициенты, характеризующие взаимодействие твердых частиц со стенками канала. С помощью методов аппроксимации были определены подгоночные коэффициенты Бусройда, которые в результате численных расчетов уравнений дают возможность определять динамические характеристики потоков с удовлетворительной точностью. В работе показаны изменения скорости и температуры газа и движущихся частиц на всем протяжении трассы, включая основной участок - фурму, опущенную в расплав чугуна.

Ключевые слова: трасса, фурма, расплав чугуна, двухфазные потоки, коэффициенты сопротивления и теплопередачи.

На основі теорії двофазних течій (газ - тверді частинки) розглянуто задачу руху потоків у виробничих трасах для подачі сипучого магнію в розплав чавуну. Дана робота в якійсь мірі підсумовує певний етап, як лабораторних експериментальних досліджень, досліджень в промислових умовах, так i теоретичних розробок. Незважаючи на великі успіхи і широке використання теорії взаємопроникливих середовищ, що є основною ідеєю підходу до розрахунку багатофазних течій і встановлення необхідних залежностей при взасмодії фаз, важливою умовою залишасться питання про силові дії стінок каналу на рухомі частинки. Наявні літературні дані дозволяють робити оцінки корисних властивостей, проте для довгих виробничих трас 3 мінливими геометичними характеристиками не знімається необхідність експериментального визначення цих параметрів в конкретних умовах.

Елисеев В. И., Толстопят А. П., Флеер Л. А., Совит Ю. П., Шевченко А. Ф., Шевченко С. А., 2019 
Такі дані були отримані в результаті багаторічної роботи на виробничих майданчиках Китаю, вони увійшли в відповідні коефіцієнти, що характеризують взасмодію твердих частинок зі стінками каналу. За допомогою методів апроксимації були визначені коефіцієнти Бусройда, які в результаті чисельних розрахунків рівнянь дають можливість визначати динамічні характеристики потоків 3 задовільною точністю. У роботі показано зміни швидкості і температури газу і рухомих частинок на всьому протязі траси, включаючи основну ділянку фурму, опущену в розплав чавуну.

Ключові слова: траса, фурма, розплав чавуну, двофазні потоки, коефіцієнти опору i теплопередачі.

On the basis of the theory of two-phase flows (gas - solid particles), the problem of the movement of flows in the production routes for the supply of bulk magnesium into the pig iron melt is considered. This work is to some extent summarizing a certain stage, as laboratory experimental research, research in industrial conditions, as well as theoretical developments. Despite the great success and wide use of the theory of interpenetrating media, which is the main idea of the approach to calculating multiphase flows, and establishing the necessary dependencies in the interaction of phases, an important condition remains the question of the force effect of the channel walls on moving particles. The available literature data allow us to make estimates of the necessary parameters, however, for long production routes with varying geometric characteristics, the need for an experimental determination of these parameters in specific conditions is not removed. Such data were obtained as a result of many years of work at the production sites of China; they were included in the corresponding coefficients characterizing the interaction of solid particles with the channel walls. Using approximation methods, Busroyd coefficients were determined, which, as a result of numerical calculations of equations, make it possible to determine the dynamic characteristics of flows with satisfactory accuracy. The work shows the changes in the velocity and temperature of the gas and moving particles along the entire length of the route, including the main section- the tuyere, lowered into the molten iron.

Key words: highway, lance, iron melt, two-phase flows, resistance coefficients and heat transfer.

Введение. Начиная с 60-70-х годов прошлого столетия научной школой Института черной металлургии были разработаны технологические методы и оборудование для эффективной десульфурации чугуна в крупногабаритных ковшах. За эти годы с помощью экспериментальных и теоретических методов исследования, a также большого накопленного опыта производственной работы, практически удалось разработать эффективную и надежную технологию, обеспечивающую высокую степень десульфурации $[1,2]$. Осуществление этого процесса стало возможным с помощью вдувания гранулированного магния через газотранспортные каналы в расплавленный металл. Однако в условиях возрастающей конкуренции и в связи с необходимостью получения глубокой десульфурации, связанной с большими расходами подаваемого магния, возникает ряд вопросов, требующих глубоких теоретических проработок, в частности, по динамике двухфазных потоков в технологической трассе, состоящей из фурмы и подводящих шлангов. Данная работа, подытоживающая в какой-то степени некоторый этап исследований, направлена на обоснование определенного метода решения этой задачи.

Постановка задачи и основные уравнения. Для изучения и расчетов параметров течения газа с твердыми частицами используется теория многофазных потоков. Она получила свое развитие во второй половине прошлого века в работах многих ученых и в настоящее время имеет довольно обширную литературу, например [3-5]. Наиболее полное представление она получила в работах Р. И. Нигматулина для газожидкостных потоков для случая бесстолкновительной монодисперсной среды, [4]. Результаты этой работы в полной мере можно использовать и в случае течения достаточно плотных дисперсных потоков при определенных условиях и допущениях. Для одномерного двухфазного стационарного потока система уравнений приобретает следующий вид: 


$$
\begin{aligned}
& \alpha_{G} \rho_{G} u_{G} S_{T}=G_{G}=\text { Const } \\
& \alpha_{C} \rho_{C} u_{C} S_{T}=G_{C}=\text { Const, } \\
& G_{G} \frac{d u_{G}}{d x}=-\alpha_{G} S_{T} \frac{d p}{d x}+\alpha_{G} 2 \pi R_{T} \tau_{G}-\alpha_{G} n S_{T}\left(f_{m}+f_{\mu}\right)+\alpha_{G} \rho_{G} S_{T} g \operatorname{Cos} \vartheta \\
& G_{C} \frac{d u_{C}}{d x}=-\alpha_{C} S_{T} \frac{\partial p}{\partial x}+\alpha_{C} 2 \pi R_{T} \tau_{C}+\alpha_{G} n S_{T}\left(f_{m}+f_{\mu}\right)+\alpha_{C} \rho_{C} S_{T} g \operatorname{Cos} \vartheta \\
& G_{G} \frac{d\left(c_{P} T_{G}\right)}{d x}=G_{G} \frac{d p}{\rho_{G} d x}+n S_{T} q_{G C}+2 \pi R_{T} \alpha_{G} q_{G}+\alpha_{G} n S_{T}\left(f_{m}+f_{\mu}\right)\left(u_{G}-u_{C}\right) \\
& G_{C} \frac{d\left(c T_{C}\right)}{d x}=G_{C} \frac{d p}{\rho_{C} d x}-n S_{T} q_{G C}+2 \pi R_{T} \alpha_{C} q_{C}
\end{aligned}
$$

где $x$ - продольная координата; $\alpha_{G}, \alpha_{C}$ - объемные доли фаз; $\rho_{G}, \rho_{C}$ - плотности; $u_{G}, u_{C}$ - проекции скоростей соответствующих фаз; $p$ - давление; $n$ концентрация дисперсной фазы; $f_{m}$ - проекция силы в продольном направлении, связанная с присоединенной массой; $f_{\mu}$ - проекция силы вязкого межфазного взаимодействия; $g$ - ускорение свободно падающего тела; $T_{G}, T_{C}$ - температуры фаз; $q_{G C}-$ межфазные тепловые потоки; $q_{G}, q_{C}-$ тепловые потоки от стенки к фазам; $S_{T}$ - площадь сечения цилиндрической трубы; $R_{T}$ - радиус трубы; $G_{G}, G_{C}-$ расходы, соответственно, газа и дисперсной среды; $c$ - теплоемкость материала частиц; $c_{P}$ - теплоемкость газа при постоянном давлении; $\tau_{G}, \tau_{C}-$ напряжения на стенке для газа и дисперсного материала. Необходимо отметить, что все параметры рассматриваются, как усредненные.

Эта система записана в предположении, что между дисперсной и дисперсионной средами отсутствует массообмен; кроме того, отброшены диссипативные и пульсационные члены для несущей среды, так как предполагается, что энергия межфазового взаимодействия значительно превосходит эти энергетические потери. К выписанным уравнениям необходимо добавить уравнение состояния газа

$$
p=\rho_{G} R_{G} T_{G},
$$

где $R_{G}$ - газовая постоянная газа-носителя.

Выпишем теперь величины в правых частях уравнений (3-6), определяющие течение рассматриваемой среды. Используем для этого хорошо известные формулы, описывающие процессы взаимодействия однородных сред с твердыми поверхностями каналов. Для расчетов величины $\tau_{G}$ для турбулентных потоков получили широкое применение формулы вида $[6,7]$

$$
\begin{aligned}
& \tau_{G}=\frac{1}{8} \xi \rho_{G} u_{G}^{2}, \quad \xi=0.316 R e_{T}^{-1 / 4} \quad \text { при } R e_{T} \leq 10^{4}, R e_{T}=\frac{\rho_{G} u_{G} R_{T}}{\mu_{G}} ; \\
& \xi=\left(1.82 \lg R e_{T}-1.64\right)^{-2} \text { при } 10^{4} \leq R e_{T} \leq 5 \cdot 10^{6} \text {, }
\end{aligned}
$$

где $\mu_{G}$ - динамический коэффициент вязкости газа; $R e_{T} \quad-$ число Рейнольдса для трубы. Для определения теплообмена газа со стенкой трубы имеются также хорошо апробированные формулы, например [7]: 


$$
\begin{gathered}
q_{G}=\alpha_{T}\left(T_{T}-T_{G}\right), \alpha_{T}=\frac{\lambda_{G}}{2 R_{T}} N u_{T}, \\
N u_{T}=\frac{\xi e_{T} P r_{G}}{8 k+12.7 \sqrt{8 \xi}\left(P r_{G}^{1 / 2}-1\right)}, \quad k=1+900 / R e_{T} \quad, \quad \operatorname{Pr}=\frac{c_{P} \mu_{G}}{\lambda_{G}},
\end{gathered}
$$

где $\alpha_{T}$ - коэффициент теплоотдачи стенки; $\lambda_{G}$ - коэффициент теплопроводности газа, $N u_{T}$ - число Нуссельта для канала; $P r_{G}$ - число Прандтля для газа.

Межфазовую силу, согласно [2], определим следующим образом:

$$
\begin{gathered}
f_{\mu}=\frac{1}{2} c_{\mu} \rho_{G} \pi R_{C}^{2}\left|u_{G}-u_{C}\right|\left(u_{G}-u_{C}\right), \\
c_{\mu}=c_{\mu}^{*}=\frac{24}{R e_{G C}}+\frac{4.4}{R e_{G C}^{1 / 2}}+0.42, \alpha_{C} \leq 0.08, \\
c_{\mu}=c_{\mu}^{* *}=\frac{4}{3 \alpha_{G}}\left(1.75+\frac{150 \alpha_{C}}{\alpha_{G} R e_{G C}}\right), \alpha_{C} \geq 0.45, \\
c_{\mu}=\frac{\left(\alpha_{C}-0.08\right) c_{\mu}^{* *}+\left(0.45-\alpha_{C}\right) c_{\mu}^{*}}{0.37},
\end{gathered}
$$

а силу присоединенной массы

$$
f_{m}=\frac{2}{3} \pi R_{C}^{3} \rho_{G}\left(\frac{d u_{G}}{d t}-\frac{d u_{C}}{d t}\right)
$$

В [3] сила присоединенных масс в канале определяется в виде

$$
\mathrm{F}_{\mathrm{m}}=\alpha_{\mathrm{G}} \mathrm{nf}_{\mathrm{m}}=\frac{1}{2} \alpha_{\mathrm{G}} \alpha_{\mathrm{C}} \rho_{\mathrm{G}}\left[\left(2 \mathrm{u}_{\mathrm{C}}-\mathrm{u}_{\mathrm{G}}\right) \frac{\mathrm{du}_{\mathrm{C}}}{\mathrm{dx}}-\mathrm{u}_{\mathrm{G}} \frac{\mathrm{du}_{\mathrm{G}}}{\mathrm{dx}}+2 \mathrm{u}_{\mathrm{C}}\left(\mathrm{u}_{\mathrm{C}}-\mathrm{u}_{\mathrm{G}}\right)\left(\frac{\mathrm{d} \alpha_{\mathrm{C}}}{\alpha_{\mathrm{C}} \mathrm{dx}}-\frac{\mathrm{dS}}{\mathrm{Sdx}}\right)\right]
$$

В [6] рекомендуется для частиц неправильной формы вместо коэффициента 0.42 в формуле (10) для $c_{\mu}$ использовать значение 0.5 , что и было принято в расчетах. Также, пользуясь данными [4], будем иметь для межфазного теплового потока

$$
\begin{gathered}
q_{G C}=4 \pi R_{C}^{2} \alpha_{G C}\left(T_{G}-T_{C}\right), \quad \alpha_{G C}=\frac{\lambda_{G}}{2 R_{C}} N u_{C} \\
N u_{C}=2+0.6 \operatorname{Re}_{G C}^{1 / 2} \operatorname{Pr}_{G}^{1 / 3}, \\
R e_{G C}=2 \frac{\rho_{G} R_{C}\left|u_{G}-u_{C}\right|}{\mu_{G}}
\end{gathered}
$$

где $\alpha_{G C}$ - коэффициент теплоотдачи; $N u_{C}$ - число Нуссельта для частицы, $R e_{G C} \quad$ число Рейнольдса для частицы, $R_{C}$ - радиус частицы. Значительную трудность в определении представляет величина $q_{C}[3]$, которая в значительной степени зависит от морфологии поверхностей канала и частиц, а также от локальных турбулентных пульсаций. В нашем случае, учитывая, что величина $\alpha_{C}$ сравнительно небольшая, а стенки и частицы являются металлическими, то есть имеют хорошую теплопроводность, в данной работе отбросим этот член в уравнении (12).

Остановимся теперь на величине $\tau_{C}$, которая играет важную роль в динамике двухфазного потока и в значительной степени определяет выходные скорости газа и частиц из фурмы. Для пневмо- и гидротранспортных систем эта 
величина представляет большой интерес, и ей посвящено большое количество работ, например [3, 8-10], в которых с различной степенью детализации показано значение этой величины в процессе транспортирования сыпучей массы. В этих работах показано, что столкновение со стенкой канала вызывается турбулентными пульсациями. Экспериментальный материал, касающийся непосредственно рассматриваемой фурмы, но для одиночной частицы, представлен в работе [11], откуда следует, что даже для прямого участка трубы для нисходящего потока потери в скорости частиц при соударение их со стенкой довольно значительны. В [12] при расчете скорости одиночной частицы в газовом потоке для $\tau_{C}$ используются формулы, выведенные в [8], которые по своей форме близки к представлению, данному в [10] для вертикальных восходящих потоков. Полученные в [12] результаты расчетов и сравнение их с экспериментальными данными показывают неплохое качественное и количественное соответствие. В целом, величину $\tau_{C}$ представим в виде суммы

$$
\tau_{C}=\tau_{C}^{W}+\tau_{C}^{G}
$$

где, согласно [8],

$$
\tau_{C}^{G}=\frac{1}{2} B_{G} \rho_{C} \frac{u_{C}}{u_{G}} \frac{R_{T}}{R_{C}} g
$$

а $\tau_{C}^{W}$ примем в виде

$$
\tau_{C}^{W}=\frac{1}{2} B_{W} \rho_{C} u_{G} u_{C} \frac{R_{T}}{R_{C}}
$$

где $B_{G}, B_{W}$ - коэффициенты Бусройда, подлежащие определению из эксперимента.

Граничные условия (начальные условия при $x=0$ ) должны задаваться из естественных соображений, однако они не всегда известны, в частности, скорость газа и скорости частиц. В нашем случае задаваемыми величинами будем считать $G_{G}$, $G_{C}, R_{T}, R_{C}$ и $p_{L}$ - давление на конце трубы. В начальной точке с хорошей степенью вероятности можно принять, что температура газа и температура частиц равны атмосферной температуре. Скорость газа и скорости частиц при $x=0$ можно получить при задании, например, $\alpha_{C}$. К сожалению, для данного процесса, начальная величина $\alpha_{C}$ или скорость частиц играют важную роль, вследствие чего эти значения довольно существенно могут повлиять на определяемые параметры и, в частности, на определение коэффициента Бусройда. Согласно [9], большую долю в общих потерях давления в трубе занимают потери на разгон сыпучей среды до некоторой равновесной скорости. Это, в основном, относится к восходящим потокам, но, как показывают расчеты, это справедливо и в нашем случае. Учитывая, что трасса довольно длинная, и она имеет повороты и сужения, которые вносят существенные сопротивления, будем считать, что начальные условия для $\alpha_{C}$ не столь важны, однако в разумных пределах. Вследствие сказанного примем эту величину ближе к значению в плотном слое: $\alpha_{C}=0,4$. Далее в наших расчетах для нахождения коэффициентов $B_{G}, B_{W}$ используем экспериментальные данные, полученные при измерении перепадов давления, как в лабораторных условиях, так и в промышленных.

Обработка лабораторных экспериментальных данных. Сравним результаты расчетов с лабораторными экспериментальными данными. В качестве 
твердой фазы были использованы частицы окатанного магния средним диаметром 1,5 мм. Эксперименты были проведены для следующих случаев:

- труба внутренним диаметром 8 мм и длиной 1,76 м при расходах несущего газа и твердой фазы:

$\mathrm{Qg}=21 \mathrm{~m}^{3} /$ час, $\mathrm{Gc}=1,565$ кг/мин;

$\mathrm{Qg}=24,75 \mathrm{~m}^{3} /$ час, $\mathrm{Gc}=1,565$ кг/мин;

$\mathrm{Qg}=30 \mathrm{~m}^{3} /$ час, $\mathrm{Gc}=1,823$ кг/мин;

- труба внутренним диаметром 10 мм и длиной 1,9 м при расходах несущего газа и твердой фазы:

$$
\begin{gathered}
\mathrm{Qg}=21 \mathrm{~m}^{3} / \mathrm{чаc}, \mathrm{Gc}=1,667 \text { кг/мин; } \\
\mathrm{Qg}=24,75 \mathrm{~m}^{3} / \mathrm{чаc}, \mathrm{Gc}=1,8825 \text { кг/мин; } \\
\mathrm{Qg}=30 \mathrm{~m}^{3} / \mathrm{чаc}, \mathrm{Gc}=2,201 \kappa г / \mathrm{мин;}
\end{gathered}
$$

- труба внутренним диаметром 13 мм и длиной 2,1 м при расходах несущего газа и твердой фазы:

$$
\begin{gathered}
\mathrm{Qg}=21 \mathrm{~m}^{3} / \text { час, Gc=4,895 кг/мин; } \\
\mathrm{Qg}=24,75 \mathrm{~m}^{3} / \text { час, Gc=4,352 кг/мин; } \\
\operatorname{Qg}=30 \mathrm{~m}^{3} / \text { час, Gc=3,396 кг/мин. }
\end{gathered}
$$

Для указанных режимов были рассчитаны теоретические перепады давлений при различных задаваемых коэффициентов Бусройда. Из расчетов и экспериментов следует, что значения коэффициентов Бусройда зависят от диаметра трубы и несколько слабее от расхода газовой и твердой фаз (возможно, что это связано со сравнительно узким интервалом изменения расходов газа и твердого материала, используемых в эксперименте). В целом эти значения находятся в диапазоне $\lg \mathrm{B}_{\mathrm{W}} \approx-(4,7-3,6)$.

Если поставить в соответствие значения коэффициентов Бусройда и диаметры труб

$$
\begin{array}{cc}
8 \mathrm{MM} & \lg \mathrm{B}_{\mathrm{W}}=-4.65 \\
10 \mathrm{MM} & \lg \mathrm{B}_{\mathrm{W}}=-3.8 \\
13 \mathrm{MM} & \lg \mathrm{B}_{\mathrm{W}}=-3.6
\end{array}
$$

то можно получить приближенное соотношение, характеризующее изменение коэффициента $B_{W}$ при переходе из одного участка трубы с радиусом $R_{T 1}$ в другой с радиусом $R_{T 2}$

$$
\lg B_{W 2}=\left(R_{T 1} /_{R_{T 2}}\right)^{0.5} \lg B_{W 1} .
$$

Это соотношение использовалось в дальнейших расчетах.

\section{Расчет промышленной трассы. Сравнение с результатами} экспериментов. На рис. 1 показана схема подводящей трассы и фурмы, для которой проводился расчет перепада давления, скоростей газа и твердых частиц магния. Для начала была рассмотрена подводящая трасса, для которой были определены коэффициенты $B_{G}, B_{W}$. 


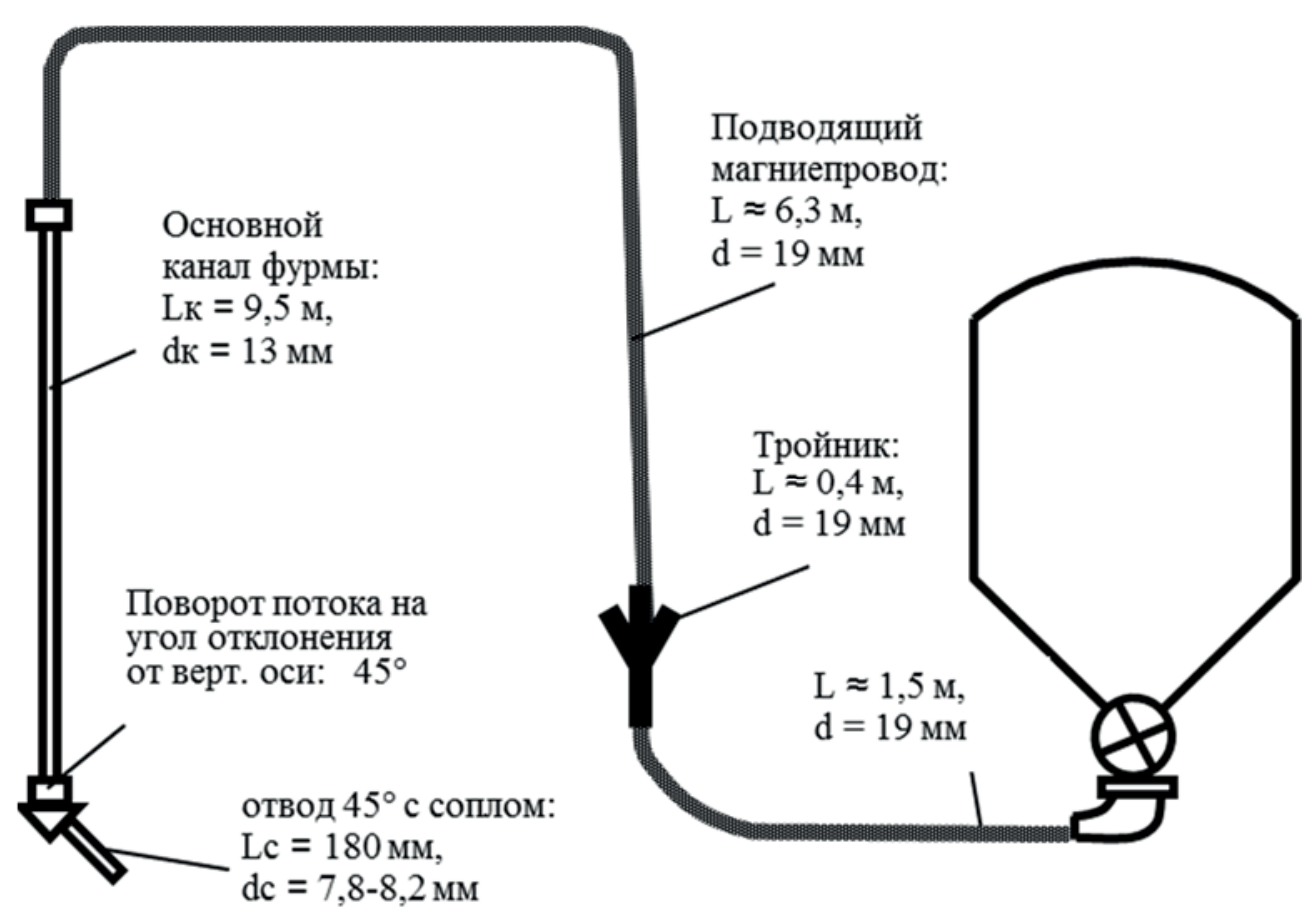

Рис. 1. Схема подводящей трассы и фурмы

Определение этих коэффициентов проводилось следующим образом: для одного варианта ( $Q=90 \mathrm{~m}^{3} /$ час, $G_{C}=20$ кг/мин и $\Delta p=0,258$ ати) при разных величинах $B_{G}$ подбирался коэффициент $B_{W}$. Затем при этих коэффициентах просчитывались все варианты расходов газа и твердого материала (некоторые из них приведены в таблице 1), относящиеся к трассе без фурмы.

Таблица 1.

Перепады давления бункер-срез сопла (МПА) при инжектировании зернистого магния на установке десульфурации чугуна Циндаоского МК при использовании различных конструкций фурменных устройств

\begin{tabular}{|c|c|c|c|c|c|}
\hline & днтенсивност & & & & \\
\hline & 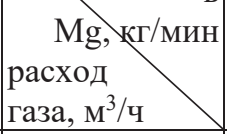 & 0 & 3 & 5 & 6 \\
\hline \multirow{3}{*}{$\begin{array}{c}\text { Фурма "С" } \\
\text { односопловая с } \\
\text { изгибом } 45^{\circ},(\varnothing \\
\text { сопла-отвода }=8 \text { мм, } \\
\text { длина } 180 \text { мм }) \\
\end{array}$} & 60 & 0,154 & & 0,300 & \\
\hline & 90 & 0,270 & & 0,435 & \\
\hline & 120 & 0,377 & & 0,555 & \\
\hline $\begin{array}{c}\text { Фурма "В" } \\
\text { односопловая с } \\
\text { изгибом } 45^{\circ},(\varnothing \\
\text { сопла-отвода }=8 \text { мм, } \\
\text { длина } 180 \text { мм }) \\
\end{array}$ & 120 & 0,365 & & & \\
\hline $\begin{array}{c}\text { Фурма "А" } \\
\text { односопловая с } \\
\text { изгибом } 45^{\circ},(\varnothing \\
\end{array}$ & 120 & 0,393 & & & \\
\hline
\end{tabular}




\begin{tabular}{|c|c|c|c|c|}
\hline $\begin{array}{c}\text { сопла-отвода }=8 \text { мм, } \\
\text { длина } 180 \text { мм) }\end{array}$ & & & & \\
\hline $\begin{array}{c}\text { Фурма "А2" } \\
\text { односопловая с } \\
\text { изгибом } 45^{\circ},(\varnothing \\
\text { сопла-отвода }=8 \text { мм, } \\
\text { длина } 180 \text { мм })\end{array}$ & 120 & 0,372 & & \\
\hline \multirow{7}{*}{$\begin{array}{c}\text { Магниепровод без } \\
\text { фурмы (Ø } 19 \text { мм, } \\
\text { длина 8,2 м) со } \\
\text { штуцером (Ø } 13 \text { мм, } \\
\text { длиною } 100 \text { мм) }\end{array}$} & 45 & 0,013 & & 0,065 \\
\hline & 60 & 0,021 & 0,081 & \\
\hline & 60 & 0,028 & 0,080 & \\
\hline & 90 & 0,043 & 0,117 & \\
\hline & 90 & 0,051 & 0,121 & \\
\hline & 120 & 0,073 & 0,159 & \\
\hline & 120 & 0,079 & 0,166 & \\
\hline \multirow{3}{*}{$\begin{array}{c}\text { Магниепровод без } \\
\text { фурмы (Ø } 19 \text { мм, } \\
\text { длина 8,2 м) без } \\
\text { штуцера }\end{array}$} & 60 & 0,019 & & \\
\hline & 90 & 0,031 & & \\
\hline & 120 & 0,044 & & \\
\hline
\end{tabular}

Далее, для всех рассчитываемых случаев определялась квадратичная ошибка. Минимум квадратичной ошибки принимался за фактическое нахождение этих величин.

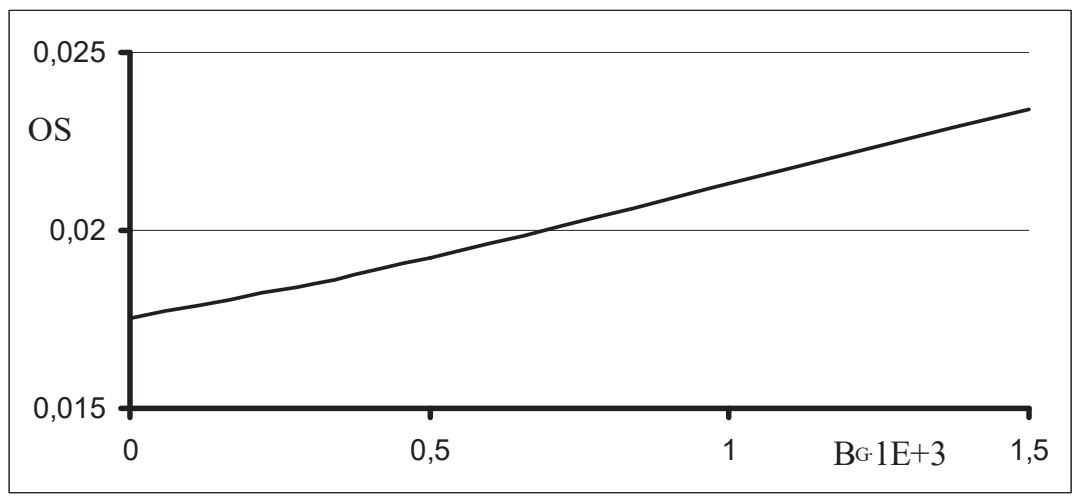

Рис. 2. Зависимость квадратичной ошибки от $B_{G}$

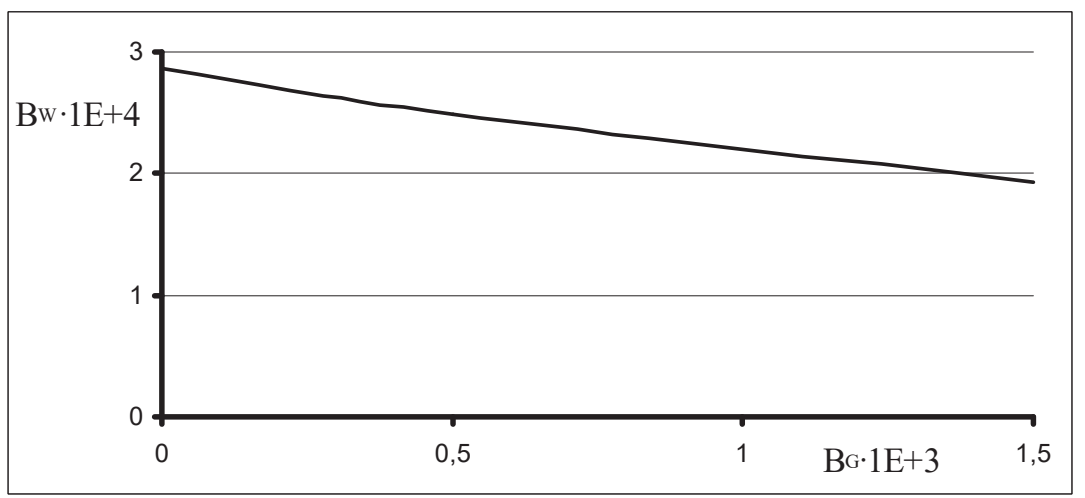

Рис. 3. Изменение $B_{W}$ от $B_{G}$ 
Эта ситуация отражена на рис. 2, 3. Из них следует, что минимум квадратичной ошибки соответствует практически значениям $B_{G}=0$ и $B_{W}=2,8669 \cdot 10^{-}$ ${ }^{4}$ или $\lg \left(2,8669 \cdot 10^{-4}\right)=-3,539$. В таблице 2 приведены экспериментальные (2-ая колонка) и теоретические расчеты (3-я колонка) перепада давления в трассе с использованием найденных коэффициентов

Перепады давлений

Таблийа 2.

\begin{tabular}{|c|c|c|c|}
\hline$Q, \mathrm{~m}^{3} / \mathrm{ч}$ & $\Delta p$ (эксп), ати & $\Delta p$ (теор), ати & $\begin{array}{l}\text { Квадратичная } \\
\text { ошибка OS (\%) }\end{array}$ \\
\hline 60 & 0,08 & 0,077 & 3,225 \\
\hline & 0,117 & 0,120 & 2,291 \\
\hline & 0,154 & 0,157 & 1,636 \\
\hline & 0,191 & 0,190 & 0,492 \\
\hline 90 & 0,121 & 0,116 & 4,140 \\
\hline & 0,171 & 0,170 & 0,708 \\
\hline & 0,215 & 0,216 & 0,530 \\
\hline & 0,258 & 0,258 & 0 \\
\hline 120 & 0,166 & 0,154 & 7,127 \\
\hline & 0,224 & 0,217 & 3,103 \\
\hline & 0,277 & 0,271 & 2,206 \\
\hline & 0,325 & 0,320 & 1,751 \\
\hline
\end{tabular}

Из последней колонки представленной таблицы следует, что результаты расчета хорошо согласуются с экспериментальными значениями.

Расчет двухфазных потоков в фурме с учетом подводящей трассы проводился по тем же выписанным выше уравнениям, при этом, так как фурма расположена вертикально, то $B_{G}=0$. Учитывая, что материал стенок канала фурмы другой, чем у шланга, величина $B_{W}$ также может отличаться от пересчитанного по формуле (23) значения с уже найденной первичной величиной $B_{W}=2,8669 \cdot 10^{-4}$. Был задан некоторый ряд значений коэффициента, близкий к рассчитанному по формуле (23), и для этого ряда значений $B_{W}$ были определены перепады давления для всех вариантов расходов газа и магния, представленных в таблице. Из этих вариантов был определен минимум квадратичной ошибки, которому соответствует значение $B_{W}=4,5 \cdot 10^{-5}$ (рис. 4).

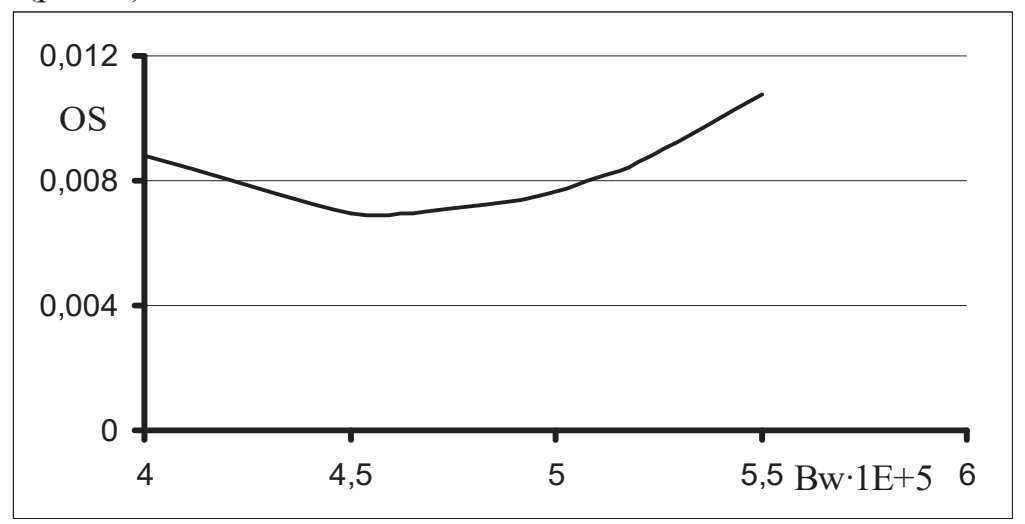

Рис. 4. Зависимость квадратичной ошибки от $B_{W}$ 
В такой постановке задачи коэффициент $B_{W}$ можно принять независимым от расхода газа. Для более точного определения коэффициента Бусройда и его дифференциации по расходам газа необходимо большее количество данных.

Приведем теперь результаты расчетов для фурмы с подводящей трассой.

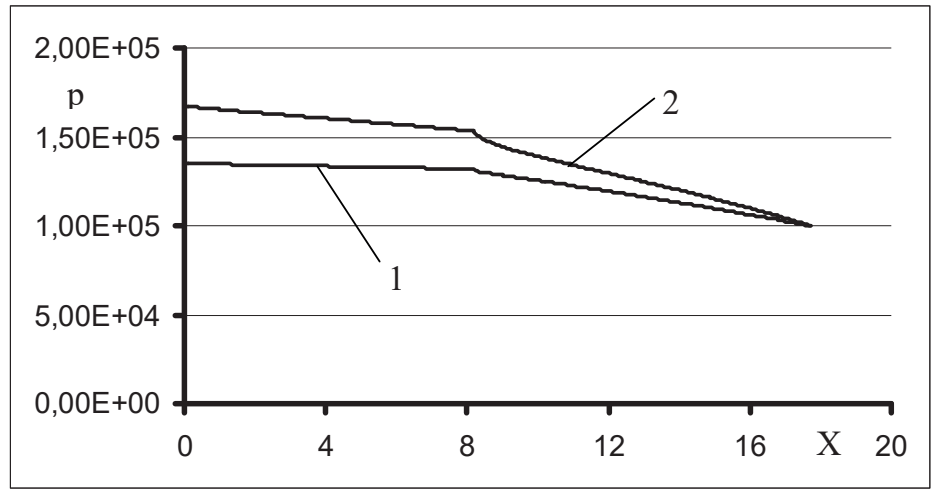

Рис. 5. Изменение давления $\mathbf{p}\left(\right.$ Н/ $\left.\mathbf{m}^{2}\right)$ вдоль трассы $\left(Q=30 \mathbf{m}^{3} /\right.$ час):

$1-\mathbf{G c}=0 ; 2-\mathbf{G c}=3$ кг/мин

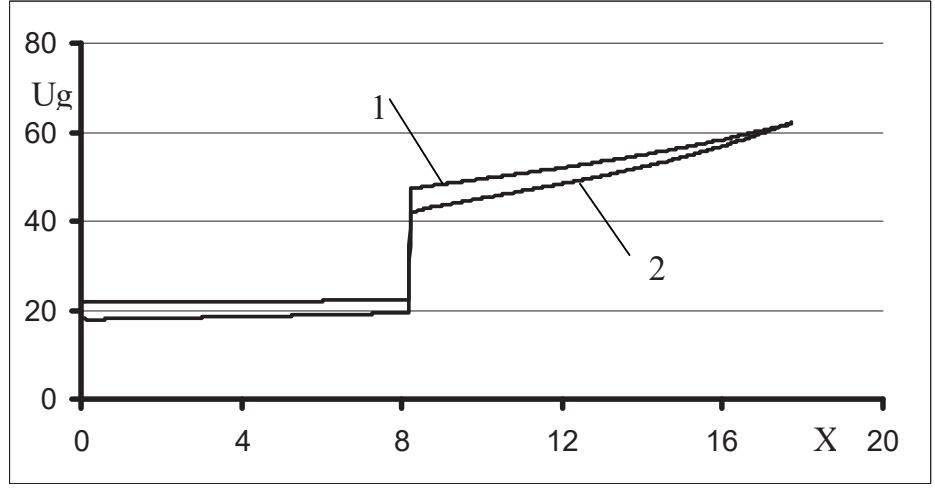

Рис. 6. Изменение скорости газа Ug (м/с) вдоль трассы ( $Q=30$ м $\left.^{3 / 4 a c}\right)$ : $1-\mathbf{G c}=0 ; 2-\mathbf{G c}=3$ кг/мин

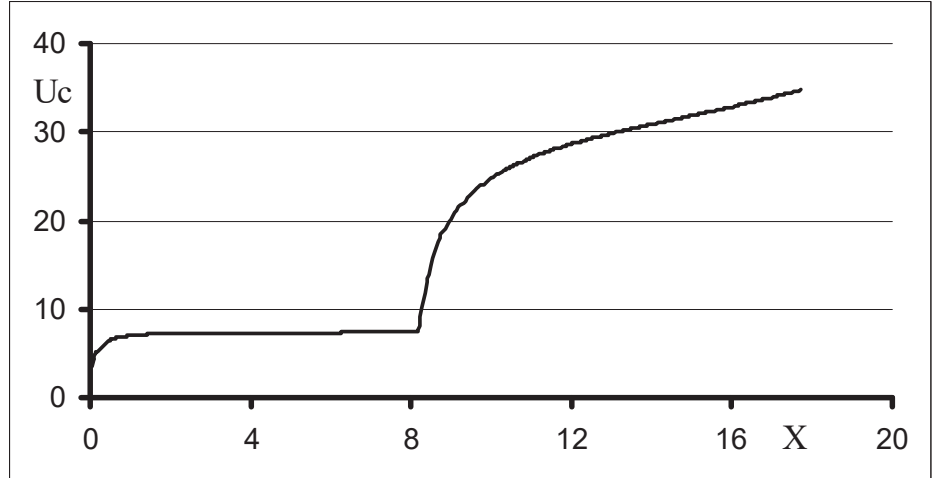

Рис. 7. Изменение скорости частиц магния Uc (м/с) вдоль трассы ( $Q=\mathbf{3 0} \mathbf{~ м}^{3} / \mathbf{4 а с}, \mathbf{G c}=\mathbf{3}$ кг/мин) 
На рис. 5 - 7 показаны кривые изменения давления (рис. 5), скорости газа (рис. 6) и скорости частиц (рис. 7) вдоль всей трассы (подводящий шланг + канал фурмы) для объемного расхода газа, равного $30 \mathrm{~m}^{3} /$ час.

На рис. 8 - 10 приведены аналогичные кривые для объемного расхода $Q$ $=60 \mathrm{~m}^{3} /$ час.

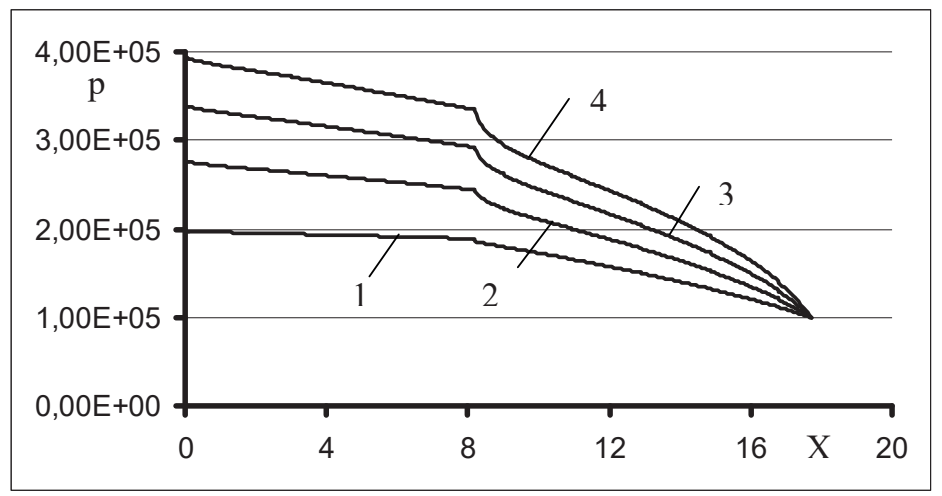

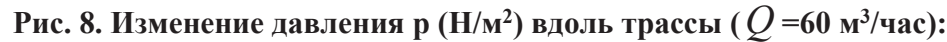
$1-\mathrm{Gc}=0 ; 2-\mathrm{Gc}=5$ кг/мин; $3-\mathrm{Gc}=10$ кг/мин; $4-\mathrm{Gc}=15$ кг/мин

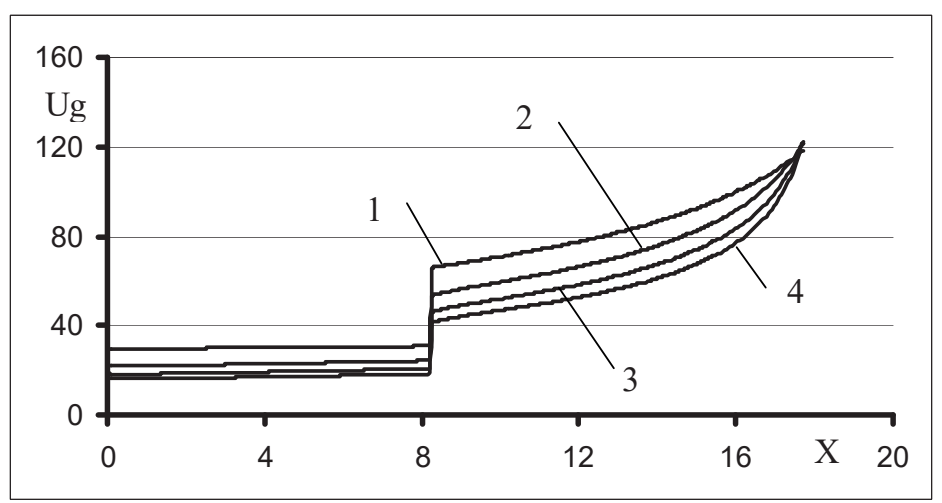

Рис. 9. Изменение скорости газа Ug (м/с) вдоль трассы $\left(Q=60 \mathrm{~m}^{3} /\right.$ час): $1-\mathrm{Gc}=0 ; 2-\mathrm{Gc}=5$ кг/мин; $3-\mathrm{Gc}=10$ кг/мин; $4-\mathrm{Gc}=15$ кг/мин

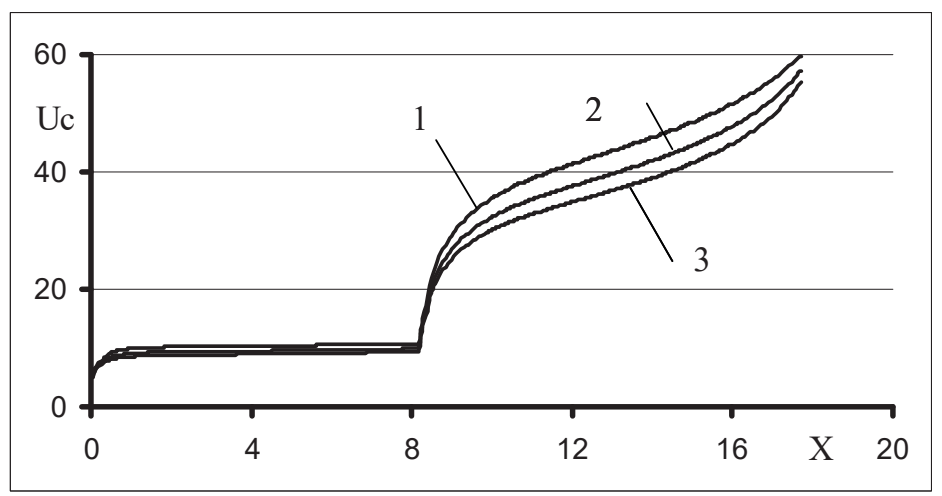

Рис. 10. Изменение скорости частиц магния Uc (м/с) вдоль трассы $\left(Q=60\right.$ м $^{3} /$ час): $1-\mathbf{G c}=5$ кг/мин; $2-\mathbf{G c}=10$ кг $/$ мин; $3-\mathbf{G c}=15$ кг $/$ мин 
На рис. 11 - 13 показаны аналогичные кривые для $Q=90 \mathrm{~m}^{3} /$ час.

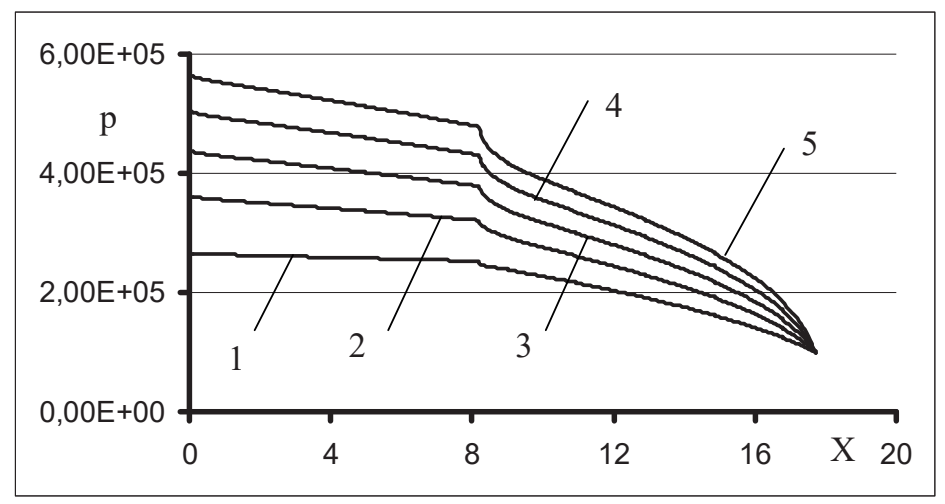

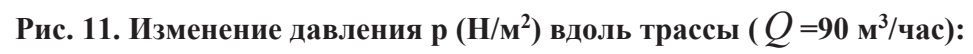

$1-\mathbf{G c}=0 ; 2-\mathbf{G c}=5$ кг/мин; $3-\mathbf{G c}=10$ кг/мин; $4-\mathrm{Gc}=15$ кг/мин; $5-\mathrm{Gc}=20$ кг/мин

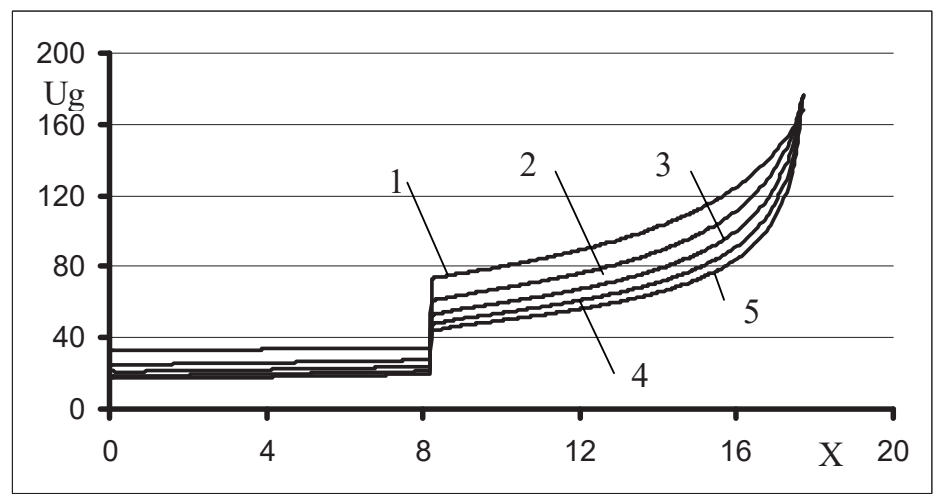

Рис. 12. Изменение скорости газа Ug (м/с) вдоль трассы ( $Q=90 \mathrm{~m}^{3} /$ час):

$$
\begin{aligned}
& 1-\mathrm{Gc}=0 ; 2-\mathbf{G c}=5 \text { кг } / \text { мин; } 3-\mathrm{Gc}=10 \text { кг } / \text { мин; } \\
& 4-\mathbf{G c}=15 \text { кг/мин; } 5-\mathbf{G c}=20 \text { кг } / \text { мин }
\end{aligned}
$$

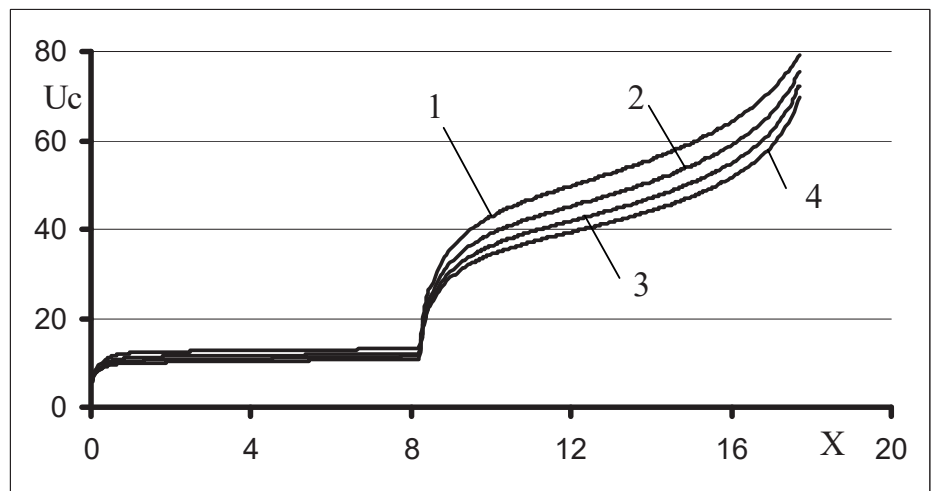

Рис. 13. Изменение скорости частиц магния Uc (м/с) вдоль трассы $\left(Q=90 \mathbf{~ м}^{3} / \mathbf{ч а с}\right)$ :

$1-\mathrm{Gc}=5$ кг/мин; $2-\mathrm{Gc}=10$ кг/мин; $3-\mathrm{Gc}=15$ кг/мин; $4-\mathrm{Gc}=20$ кг/мин 
Из этих рисунков видно, что они типовые, то есть соответствующие кривые качественно не отличаются друг от друга, хотя в количественном отношении они сильно разнятся. В первую очередь заметно, что область течения состоит из двух подобластей - это подводящая трасса и канал фурмы с существенно меньшим диаметром, чем внутренний диаметр шланга. Отсюда следует, что общий перепад давления в основном связан с сопротивлением фурмы, однако перепад давления на шланге составляет $10-15 \%$ от общего перепада. При переходе из шланга в канал хорошо заметны падения давления и резкие подъемы скоростей газа и частиц магния. Далее вдоль канала давление уменьшается, причем крутизна его изменения возрастает. Скорости газа возрастают, и у выхода из канала рост скорости становится почти вертикальным. Особенно это хорошо видно для большого расхода, т.е. для $Q$ $=90 \mathrm{~m}^{3} /$ час.

Точность расчетов и полученных параметров можно оценить по данным следующих таблиц, где показаны рассчитанные и экспериментальные перепады давления на всей трассе. В таблице 3 приведены теоретические и экспериментальные перепады давления для газа.

\section{Перепады давлений для чистого газа}

Таблиц̧а 3.

\begin{tabular}{|c|l|l|l|}
\hline$Q$ м $^{3} /$ час & $\begin{array}{l}\Delta p \text { (эксп) } \\
\text { МПА }\end{array}$ & $\begin{array}{l}\Delta p \text { (теор) } \\
\text { МПА }\end{array}$ & OS \% \\
\hline 30 & 0,037 & 0,036 & 2,703 \\
\hline 60 & 0,098 & 0,099 & $-0,612$ \\
\hline 90 & 0,175 & 0,168 & 4,229 \\
\hline 120 & 0,239 & 0,239 & 0,067 \\
\hline
\end{tabular}

В таблице 4 показаны соответствующие перепады давлений для двухфазных сред.

Перепады давлений для двухфазной среды

Таблица 4

\begin{tabular}{|c|l|l|l|}
\hline & $\begin{array}{l}\Delta p \\
\text { (эксп) } \\
\text { МПА }\end{array}$ & $\begin{array}{l}\Delta p \\
\text { (теор) } \\
\text { MПА }\end{array}$ & OS \% \\
\hline 30 & 0,079 & 0,068 & 13,797 \\
\hline 60 & 0,195 & 0,178 & 8,872 \\
\hline & 0,249 & 0,242 & 3,012 \\
\hline & 0,3 & 0,297 & 0,867 \\
\hline 90 & 0,264 & 0,264 & 0,189 \\
\hline & 0,335 & 0,341 & $-1,761$ \\
\hline & 0,404 & 0,409 & $-1,163$ \\
\hline & 0,466 & 0,471 & $-1,009$ \\
\hline
\end{tabular}

Из приведенных таблиц следует, что в целом результаты расчетов вполне удовлетворительны - как для чистого газа, так и для двухфазных потоков. Таким образом, для канала фурмы можно принять значение коэффициента Бусройда $B_{W}=4,5 \cdot 10^{-5}$, а в подводящей трассе $-B_{W}=2,8669 \cdot 10^{-4}$. 
В свою очередь в сводной таблице 4 приведены измеренные на реальном объекте перепады давления бункер-срез сопла (МПА) при инжектировании зернистого магния на установке десульфурации чугуна Циндаоского МК при использовании различных конструкций фурменных устройств

Выводы. Разработана теория движения двухфазных сред в подводящей трассе и канале фурмы при подаче гранулированного магния для десульфурации расплава чугуна. На основе лабораторных исследований и экспериментальных данных, полученных для промышленных объектов, найдены необходимые коэффициенты, замыкающие теорию. Сравнение экспериментальных и теоретических значений перепадов давления показала приемлемое согласование.

\section{Библиографические ссылки}

1. Шевченко, А. Ф. Технология и оборудование десульфурации чугуна магнием в большегрузных ковшах [Текст] / А .Ф. Шевченко, В. И. Большаков, А. М. Башмаков. - Киев: Наукова думка. 2011. - 208 с.

2. Шевченко, А. Ф. Внепечная десульфурация чугуна в ковшах. Технология, исследования, анализ, совершенствование [Текст] / А. Ф. Шевченко, И. А. Маначин, А. С. Вергун, Б. В. Двоскин, В. Г. Кисляков, С. А. Шевченко, А. В. Остапенко. - Днепр: ДніпроVAL, 2017. $-253 \mathrm{c}$.

3. Соу, С. Гидродинамика многофазных систем [Текст] / С. Соу. Под ред. М. Е. Дейча. - М.: Мир, 1971. - 536 с.

4. Нигматулин, Р. И. Динамика многофазных сред. Ч. І. [Текст] / Р. И. Нигматулин. М.: Наука, 1987. - 464 с.

5. Накорчевский, А. И. Гидродинамика и тепломассоперенос в гетерогенных системах и пульсирующих потоках [Текст] / А. И. Накорчевский, Б. И. Басок. Под ред. А. А. Долинского. - Киев: Наукова думка, 2001. - 346 с.

6. Лойцянский, Л. Г. Механика жидкости и газа: Учеб. для вузов [Текст]. / Л. Г. Лойцянский. - М.: Дрофа, 2003. - 840 с.

7. Тимошенко, В. И. Газовая динамика высокотемпературных технологических процессов [Текст] / В. И. Тимошенко. - Днепропетровск: Институт технической механики НАНУ и НКАУ, 2003. -460 с.

8. Волошин, А. И. Механика пневмотранспортирования сыпучих материалов [Текст] / А. И. Волошин, Б. В. Пономарев. - Киев: Наукова думка, 2001. - 520 с.

9. Зуев, Ф. Г. Пневматическое транспортирование на зерноперерабатывающих предприятиях [Текст] / Ф. Г. Зуев. - М.: Колос, 1976. - 344 с.

10. Шрайбер, А. А. Гидромеханика двухкомпонентных потоков с твердым полидисперсным веществом [Текст] / А. А. Шрайбер, В. Н. Милютин, В. П. Яценко. - Киев: Наукова думка, 1980. - 250 с.

11. Толстопят, А. П. Экспериментальное определение скорости частиц в канале постоянного диаметра [Текст] / А. П. Толстопят, Л. А. Флеер, В. В. Давидсон, В. И. Елисеев, Т. А. Рузова, А. Ф. Шевченко, С. А. Шевченко // Фундаментальные и прикладные проблемы черной металлургии. Сборник научных трудов. - 2011. - Вып. 23. - С. 113-122.

12. Елисеев, В. И. Расчет движения частиц в прямолинейном канале фурмы [Текст] / В. И. Елисеев, А. П. Толстопят, Л. А. Флеер, Т. А. Рузова, А. Ф. Шевченко, С. А. Шевченко // Фундаментальные и прикладные проблемы черной металлургии. Сборник научных трудов. - 2011. - Вып. 24. - С. 113-121. 